\title{
CRISPR/Cas9 - mediated genome editing of bread wheat to modulate heading time
}

\author{
Miroshnichenko D. ${ }^{1,2,3 *}$, Timerbaev V.1,2,3, Klementyeva A. ${ }^{2,3}$, Salina E. ${ }^{4}$, \\ Dolgov S. ${ }^{2,3}$ \\ ${ }^{1}$ Kurchatov Genomic Center - ARRIAB, All-Russia Research Institute of Agricultural Biotechnology, \\ Moscow, Russia \\ ${ }^{2}$ Shemyakin and Ovchinnikov Institute of Bioorganic Chemistry RAS, Pushchino, Russia \\ ${ }^{3}$ All-Russia Research Institute of Agricultural Biotechnology, Moscow, Russia \\ ${ }^{4}$ Institute of Cytology and Genetics, SB RAS, Novosibirsk, Russia \\ *email: miroshnichenko@bibch.ru
}

The heading time of spring and winter varieties largely depends on their need for vernalization and controlled by a group of coordinated $V R N$ genes (VRN1, VRN2, VRN3 and VRN4). In bread wheat the presence of at least one dominant allele of VRN1 gene homologue (Vrn-Al, Vrn-Bl, or Vrn-Dl) determines the spring growth habit; winter wheat contains only recessive alleles. Various combinations of dominant/recessive homologues can lead to differences in flowering time, height of plants and yield. The functional difference between dominant and recessive alleles associated with a presence of mutations in the promoter region or the first intron of $\mathrm{Vrn}-1$. The goal of our study was to produce wheat plants with new nucleotide changes in the conserved promoter region of the recessive $v r n-A l$ gene using genomic editing technique. We have developed an effective protocol for delivery of editing components of the CRISPR/Cas9 system into wheat calli, followed by the regeneration of genome edited plants. As a result of co-transformation by two vectors, carrying the sequence of $v r n-A l$-specific guide sgRNA and the Cas9 nuclease, a number of independent events containing various changes in the promoter region were produced. Analysis of the T1-T2 seed generation confirmed the inheritance of mutant promoter variants including single nucleotide deletion/ substitutions, and deletion of four-eight nucleotides. More detailed results concerning the efficiency of bread wheat genome editing using various types of sgRNA, the possibility to clean up the plants from 'editing tools' sequence, and the impact of genomic edits on an earing time will be presented.

Funding: Kurchatov Genomic Center of All-Russia Research Institute of Agricultural Biotechnology, agreement No. 075-15-2019-1667. 